Check for updates

Cite this: RSC Adv., 2019, 9, 529

Received 7th November 2018

Accepted 19th December 2018

DOI: $10.1039 / \mathrm{c} 8 \mathrm{ra09201j}$

rsc.li/rsc-advances

\section{Metal-organic frameworks in a blended polythiophene hybrid film with surface-mediated vertical phase separation for the fabrication of a humidity sensor $\dagger$}

\author{
Young Jin Jang, Yoo Eil Jung, Gun Woo Kim, Chang Yeon Lee (iD * \\ and Yeong Don Park (D)*
}

A facile, reliable, fast-response poly(3-hexylthiophene-2,5-diyl) (P3HT)-based humidity sensor was developed by introducing metal-organic frameworks (MOFs), HKUST-1, into the semiconducting layer. HKUST-1 displayed an excellent ability to capture water molecules, thereby generating and attracting charge carriers derived from the water molecules present in the active layer. The HKUST-1/P3HT hybrid film showed excellent device sensitivity with an enhanced electrical current and a threshold voltage shift as a function of the relative humidity due to the superior gas capture properties and the porosity of HKUST-1. The surface energy of the substrate altered the distribution and location of HKUST-1 in the active layer, which improved the sensitivity of the hydrophilic surface. A dynamic gas sensing test revealed that the hybrid film displayed a reliable and stable performance with fast response and recovery times. The introduction of MOFs into a conjugated polymer stabilized and sensitized the devices, providing a facile method of improving gas sensor technologies based on organic semiconductors.

\section{Introduction}

Organic electronics have attracted considerable interest in recent decades as the demand for portable and low-cost electronic devices has increased. ${ }^{\mathbf{1 - 5}}$ Organic thin-film transistors (OTFTs) have been investigated for various applications, including radio-frequency identification tags, display backplanes, smart cards, and sensors. ${ }^{6-8}$ OTFTs are regarded as an ideal flexible platform for creating portable, lightweight, and robust devices. A variety of signal transduction devices have been explored for use in monitoring changes in the capacitance, resistance, and impedance of organic or inorganic materials, and OTFT platforms continue to show promise in flexible sensor technologies..$^{9-13}$ OTFTs are sensitive to physical and chemical stimuli because small interactions between a semiconductor and a target analyte amplify the electrical signal of a transistor device via the field-effect mobility, drain current, and threshold voltage. ${ }^{\mathbf{1 4 - 1 7}}$ Note that analytes diffuse into the bulk of the semiconductor or insulator layer and finally arrive at the interface between the semiconductor and the gate dielectric layer. ${ }^{2,18,19}$ These analytes significantly affect the charge carriers near the interface and alter the drain current or threshold

Department of Energy and Chemical Engineering, Incheon National University, Incheon 22012, Republic of Korea. E-mail: cylee@inu.ac.kr; ydpark@inu.ac.kr

$\dagger$ Electronic supplementary information (ESI) available. See DOI: 10.1039/c8ra09201j voltage. Effective delivery of analyte molecules to the gate dielectric interface plays an important role in improving the sensing abilities of the OTFT device. ${ }^{20}$

Organic transistors inevitably suffer from poor stability and electrical properties, resulting in the slow response and recovery of the sensor device..$^{\mathbf{1 0 2 1 , 2 2}}$ We attempted to address this issue by developing an OTFT-based humidity sensor, in which a humidity-capturing material was inserted into the polymeric semiconductor (Fig. 1). Metal-organic frameworks (MOFs) are rapidly emerging as an organic/inorganic hybrid porous material that can easily self-assemble through an organic linker and a metal ion cluster. ${ }^{23-25}$ In addition to providing an excellent porosity compared to other porous materials, such as zeolites and carbon materials, the large surface area, tunable pore size, and functional diversity of MOFs lends these materials utility in (a)

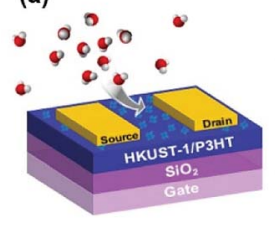

(b)

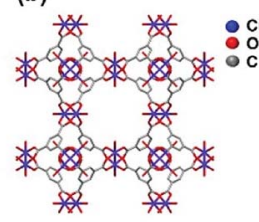

(c)

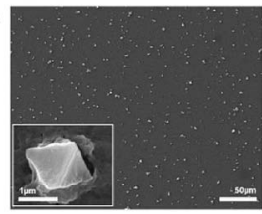

Fig. 1 (a) Schematic diagram showing the humidity sensor device based on a HKUST-1/P3HT hybrid film. (b) Schematic views of the HKUST-1 crystal structures. (c) Morphologies of the synthesized HKUST-1 coated on the hybrid film, characterized by a low and high (inset) magnification FE-SEM. 
gas storage, gas separation, catalysis, and sensing applications. ${ }^{26-29}$

In this study, we developed a OTFT-based sensor by fabricating a hybrid film blend comprising a conjugated polymer and MOFs. Among the various types of MOFs tested, the wateradsorbing HKUST-1 $\left(\mathrm{Cu}_{3}(\mathrm{BTC})_{2}, \quad\right.$ BTC $=1,3,5$-benzenetricarboxylate) was selected as a suitable material for humidity sensing due to its excellent water adsorption and promising utility in molecular sensing. ${ }^{30,31}$ As shown in Fig. 1a, a HKUST-1/ poly(3-hexylthiophene-2,5-diyl) (P3HT) hybrid film coating was applied, used as a semiconducting layer, and then exposed to a humid environment to monitor its sensing characteristics. A schematic diagram of the crystal structure of HKUST-1 is presented in Fig. 1b. The $I-V$ characteristic behavior indicated that the gas capture properties of HKUST-1 readily delivered gas molecules to the channel region, thereby enhancing the sensing properties of the device. The quantity of HKUST- 1 incorporated into the P3HT solution was varied and the location of HKUST-1 was controlled by tuning the surface energy of the substrate to induce effective current variation in the device. The hybrid humidity sensor fabricated using the HKUST-1/P3HT hybrid film displayed fast response and recovery times and a reliable stable performance toward detecting changes in the humidity levels. The present study successfully deployed MOFs in organic electronics, effectively controlling the distribution of MOFs in the active layer and altering the electrical properties. This strategy could potentially be useful in OTFT gas sensors beyond humidity sensors by using a variety of MOFs.

\section{Experimental section}

\section{Hybrid film and device fabrication}

Poly(3-hexylthiophene-2,5-diyl) (P3HT, regioregularity $=$ 91$94 \%, M_{\mathrm{w}}=28 \mathrm{kDa}$ ) was purchased from Rieke Metals Inc. A $10 \mathrm{mg}$ sample of P3HT and various amounts of HKUST-1 (1, 2, 3, and $5 \mathrm{mg}$ ) were blended in $1 \mathrm{~mL}$ tetrahydrofuran (THF, 99.8\%). To achieve an effective dispersion, each material was combined after stirring for $30 \mathrm{~min}$ at $45{ }^{\circ} \mathrm{C}$. The mixture was sonicated at $45^{\circ} \mathrm{C}$ for $1 \mathrm{~h}$ and stirred at $45^{\circ} \mathrm{C}$ for $4 \mathrm{~h}$. The solution was spincoated at $2000 \mathrm{rpm}$ for $60 \mathrm{~s}$ (Spin-1200D, Midas) onto heavily doped n-type silicon ( $\mathrm{Si}$ ) wafers covered with a $300 \mathrm{~nm}$ thick silicon dioxide $\left(\mathrm{SiO}_{2}\right)$ layer. The surfaces of the $\mathrm{SiO}_{2} / \mathrm{Si}$ substrate were modified to control the surface energies using a selfassembled monolayer. The $\mathrm{OH}$ substrate was prepared by washing a Si wafer using the piranha solution $(2: 1$ mixture of $98 \%$ sulfuric acid and $35 \%$ hydrogen peroxide) for $30 \mathrm{~min}$ at $70{ }^{\circ} \mathrm{C}$ and was rinsed several times using deionized water. Hexamethyldisilazane (HMDS, Sigma-Aldrich, Inc.) was spincoated onto a cleaned Si wafer at $3000 \mathrm{rpm}$ for $30 \mathrm{~s}$ and dried overnight in a vacuum oven. Octadecyltrichlorosilane (ODTS, Sigma-Aldrich, Inc.) was coated onto a cleaned $\mathrm{SiO}_{2} / \mathrm{Si}$ substrate by dipping in toluene for $1.5 \mathrm{~h}$. The top contact device was formed by evaporating gold source/drain electrodes onto the spin-coated HKUST-1/P3HT film using a shadow mask (channel length $=100 \mu \mathrm{m}$, channel width $=2000 \mu \mathrm{m})$. In order to prevent the water or solvent molecules occupying the pores of HKUST-1 during the device fabrication process, samples were kept in a high vacuum for $6 \mathrm{~h}$. For the UV-Vis adsorption analysis, identical HKUST-1/P3HT films were prepared onto the glass substrate with the same surface treatments.

\section{Characterization}

The molecular structure of the synthesized HKUST-1 was characterized by X-ray diffraction (Rigaku smart lab) with $\mathrm{Cu} \mathrm{K \alpha}$ radiation. $\mathrm{N}_{2}$ adsorption/desorption isotherms of HKUST-1 were obtained from Quantachrome Instruments (Boynton Beach, FL) using the Autosorb-iQ Win software package, and the specific surface areas were calculated in the linear range using the Brunauer-Emmett-Teller model. Pore size distribution of the HKUST- 1 was obtained from $\mathrm{N}_{2}$ isotherm with the nonlocal density functional theory (NLDFT) method. The HKUST-1 particle size was characterized using field-emission scanning electron microscopy (JEOL, JSM-7800F) employing an accelerating voltage of $15 \mathrm{kV}$, and Pt coating was processed prior to analysis. Optical microscopy (Olympus BX51) was used to obtain optical images of the MOF-blended polymer film. The wettability of the hybrid film surface was obtained using a contact angle meter (SEO 300A, SEO Co.). The elemental analysis was obtained using an energy dispersive spectrometer equipped with a JEOL, JSM-7800F employing an accelerating voltage of $15 \mathrm{kV}$.

X-ray photoelectron spectroscopy spectra were used to investigate the HKUST-1 distribution in a hybrid film using monochromatic Al K $\alpha$ X-ray source in a PHI 5000 Versa Probe II. The depth profile was obtained by a sequence of Ar ion gun etch and measurement and the binding energies were calibrated to the $\mathrm{C}$ 1s peak at $284.5 \mathrm{eV}$. A short etching process was performed to eliminate contamination from the sample surface. The etching process was performed in 1 minute intervals, and XPS data were collected at each depth to obtain the depth profiles of the films. Flipped samples were prepared by detaching thin films from the substrates by using epoxy adhesive. Epoxy adhesive was placed on the thin film and cured for $30 \mathrm{~min}$. After keeping under vacuum at room temperature for $12 \mathrm{~h}$, samples were annealed at $60^{\circ} \mathrm{C}$ for longer than $12 \mathrm{~h}$ under vacuum. Thin film attached with epoxy adhesive was separated from the substrate by soaking into the liquid nitrogen.

UV-Vis adsorption spectra were acquired by using a UV-Vis spectrophotometer Thermo Scientific, Genesys 10S spectrometer). Ellipsometer ((J. A. Woollam Co. Inc) was used to determine the thickness of the film. The electrical performances of the field-effect transistors were estimated using a semiconductor analyzer (Keithley 4200-SCS) at room temperature (23-25 $\left.{ }^{\circ} \mathrm{C}\right)$. Gas sensing properties were measured using a gas chamber (M5VC, MS TECH) and a Keithley 4200-SCS at room temperature. Gas mixtures of wet $\mathrm{N}_{2}$ and dry were introduced into the gas chamber. The composition of gas mixtures was tuned by mass flow controller and the relative humidity were measured by a hygrometer (Testo) which has $\pm 0.1 \%$ of accuracy. The field-effect mobility $(\mu)$ and threshold voltage $\left(V_{\mathrm{T}}\right)$ were obtained in the saturation regime according to the equation

$$
I_{\mathrm{D}}=\frac{W}{2 L} \mu C_{\mathrm{i}}\left(V_{\mathrm{G}}-V_{\mathrm{T}}\right)^{2}
$$


where $I_{\mathrm{D}}$ is the drain-current, $V_{\mathrm{G}}$ is the gate source voltage and $C_{\mathrm{i}}$ is the gate dielectric capacitance $\left(10.8 \mathrm{nF} \mathrm{cm}^{-2}\right)$.

\section{Results and discussion}

HKUST-1 crystals with 0.9-1.4 $\mu \mathrm{m}$ were successfully synthesized and were characterized, as shown in Fig. S1, $\dagger$ and the HKUST-1/ P3HT hybrid film was confirmed to feature fluorescent HKUST1 crystals, as evidenced using polarized light microscopy images, shown in Fig. S2. $\dagger^{32,33}$ HKUST-1 crystals were embedded in $70-90 \mathrm{~nm}$ thick of P3HT film and spaced several tens of micrometers apart. Fig. 2 shows the transfer characteristics of a HKUST-1/P3HT hybrid thin film at a fixed drain voltage, $V_{\mathrm{D}}=$ $-60 \mathrm{~V}$ and a relative humidity of $0 \%$ to $30 \%$ at room temperature. Various amounts of HKUST-1 $(0,1,2,3$, or $5 \mathrm{mg})$ were added to the P3HT solution (10 $\mathrm{mg} \mathrm{mL}^{-1}$ ), and HKUST-1/P3HT films were fabricated to investigate the electrical current change. As the relative humidity increased, the overall drain current level increased and the threshold voltage shifted toward positive values in all OTFT devices. As the relative humidity returned to $0 \%$, the transfer characteristics returned to their initial curve. In a pristine P3HT film, the drain current level at $V_{\mathrm{G}}=25 \mathrm{~V}$ increased slightly, and the threshold voltage shifted below $5 \mathrm{~V}$ in the positive direction as the relative humidity changed from $0 \%$ to $30 \%$ (see Fig. 2a). The on-current level and the field-effect mobility decreased as the amount of HKUST-1 increased because the non-conducting properties of HKUST-1 deteriorated the charge carrier transport. The electrical conductivity in the pristine P3HT film measured by 2-point probe was $3.23 \times 10^{-3} \mathrm{~S} \mathrm{~cm}^{-1}$ but the electrical conductivity value in the $2 \mathrm{mg} \mathrm{mL}^{-1}$ HKUST-1 blended P3HT film decreased to $7.53 \times 10^{-4} \mathrm{~S} \mathrm{~cm}^{-1}$. The drain current level $\left(I_{30} / I_{0}\right)$ and the threshold voltage $\left(\Delta V_{\text {th }}\right)$ varied sensitively as the humidity changed between 0 and 30\% in the HKUST-1/P3HT hybrid film. In the P3HT film prepared with $3 \mathrm{mg} \mathrm{mL}^{-1}$ HKUST-1, $I_{30} / I_{0}$ at $V_{\mathrm{G}}$ $=25 \mathrm{~V}$ varied by a factor of 80 and the threshold voltage shifted by $40 \mathrm{~V}$, which is a surprisingly large value compared with the value obtained from the pristine P3HT thin films (see Fig. 2a-e). The values of $I_{30} / I_{0}, \Delta V_{\mathrm{th}}$, and the field-effect mobility are listed for all fabricated devices in Table 1. MOFs have an outstanding surface area and gas adsorption properties, and they attract numerous numbers of water molecules in the active layer, resulting in significant changes in the electrical properties, even in devices with low HKUST-1 contents. The sensitivity of the HKUST-1/P3HT film increased as the HKUST-1 content increased, but decreased above $3 \mathrm{mg} \mathrm{mL}{ }^{-1}$ HKUST- 1 in the blended film (Fig. 2f). Quantities of HKUST-1 beyond $3 \mathrm{mg}$ $\mathrm{mL}^{-1}$ displayed HKUST-1 agglomeration, as shown in Fig. S3. $\dagger$ HKUST-1 agglomeration reduces the available surface area and the number of analytes that can be detected, thereby diminishing the sensitivity of the device. Unlike most of the polymerbased humidity sensors which provide moderate device sensitivity at high relative humidity level, HKUST-1/P3HT blended film show outstanding device sensitivity at environmental condition. ${ }^{34-36}$ The response of HKUST-1/P3HT hybrid film at a wide-range of relative humidity is shown in Fig. S4. $\dagger$ As the relative humidity increases, the variation of the electrical current increased, but the variation slope to the relative humidity at over $30 \%$ decreased. Therefore, in this study, our (a)

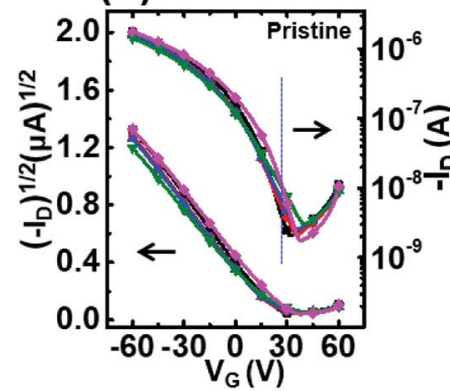

(d)

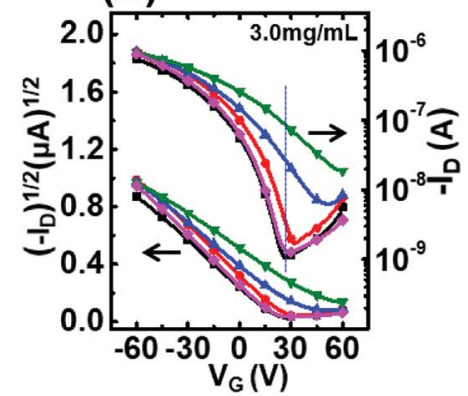

(b)

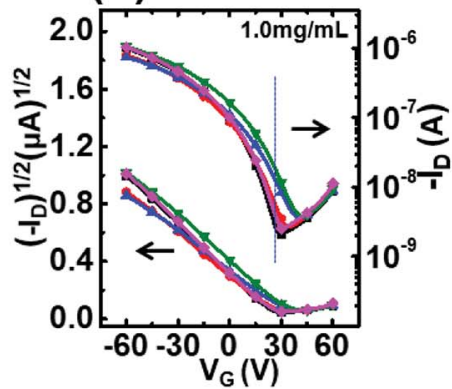

(e)

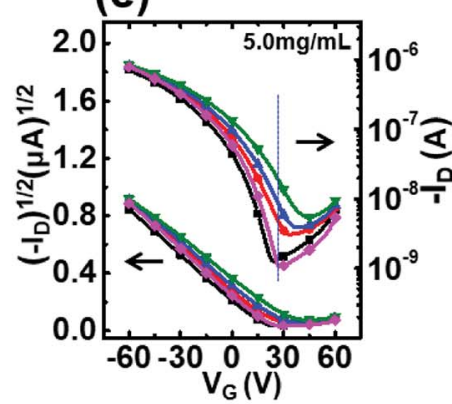

(c)

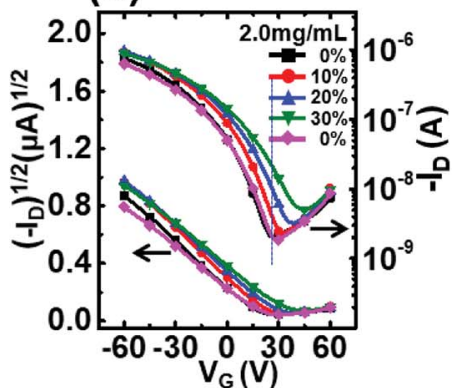

(f)

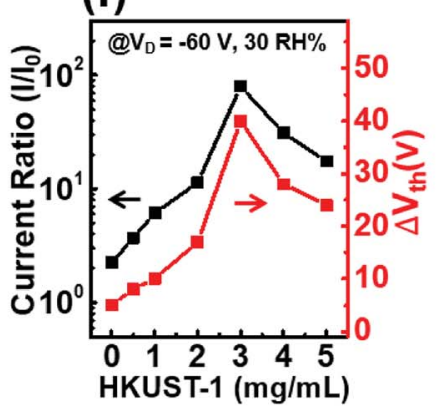

Fig. 2 Transfer characteristics $\left(I_{D}-V_{G}\right)$ of the HKUST-1/P3HT hybrid film at a fixed drain voltage $\left(V_{D}=-60 \mathrm{~V}\right)$ over relative humidity values of 0 to $30 \%$. The hybrid film was coated onto HMDS substrates, and various amounts of HKUST-1s were added to the $10 \mathrm{mg} \mathrm{mL}^{-1} \mathrm{P} 3 \mathrm{HT}$ solution: (a) pristine polymer film, (b) $1 \mathrm{mg}$, (c) $2 \mathrm{mg}$, (d) $3 \mathrm{mg}$ and (e) $5 \mathrm{mg}$. (f) Summary of the current ratios at $V_{\mathrm{G}}=25 \mathrm{~V}$ and the threshold voltages as a function of the HKUST-1 content. RHO\% indicates re-measured value when the relative humidity returns to $0 \%$. 
Table 1 Summary of the performances of the HKUST-1/P3HT films prepared with various HKUST-1 concentrations on an HMDS substrate

\begin{tabular}{|c|c|c|c|c|}
\hline $\begin{array}{l}\text { Concentration } \\
\left(\mathrm{mg} \mathrm{mL}^{-1}\right)(\%)\end{array}$ & $\mu_{\mathrm{RH} 0 \%}{ }^{a}\left(\mathrm{~cm}^{2} \mathrm{~V}^{-1} \mathrm{~s}^{-1}\right)$ & $\mu_{\mathrm{RH} 30 \%}{ }^{b}\left(\mathrm{~cm}^{2} \mathrm{~V}^{-1} \mathrm{~s}^{-1}\right)$ & $\Delta V_{\mathrm{th}}^{c}(\mathrm{~V})$ & $I_{\mathrm{RH} 30 \%} / I_{\mathrm{RH} 0 \%}{ }^{d}$ \\
\hline $0.0(0 \%)$ & $2.2 \times 10^{-3}$ & $1.6 \times 10^{-3}$ & 4 & 2.0 \\
\hline $2.0(20 \%)$ & $1.0 \times 10^{-3}$ & $9.4 \times 10^{-4}$ & 17 & 11.4 \\
\hline $3.0(30 \%)$ & $1.0 \times 10^{-3}$ & $6.5 \times 10^{-4}$ & 40 & 80.0 \\
\hline $4.0(40 \%)$ & $1.0 \times 10^{-3}$ & $8.2 \times 10^{-4}$ & 28 & 31.1 \\
\hline
\end{tabular}

${ }^{a}$ Average field-effect mobility calculated in the saturation regime at $0 \%$ relative humidity. ${ }^{b}$ Average field-effect mobility calculated in the saturation regime at $30 \%$ relative humidity. ${ }^{c}$ The variations in the threshold voltage shift. ${ }^{d}$ Current ratio of $I_{\mathrm{D}}$ at a $30 \%$ relative humidity compared to $I_{\mathrm{D}}$ at a $0 \%$ relative humidity, at $V_{\mathrm{G}}=25 \mathrm{~V}$.

researches focus on the device test at a relative humidity of $0 \%$ to $30 \%$.

The substrate surface energy was modified to control the HKUST-1 distribution in the P3HT film and the film's electrical properties. To this end, the substrate surface was treated with self-assembled monolayers comprising a hexamethyldisilazane (HMDS) and octadecyltrichlorosilane (ODTS) coupling agent. For comparison, a $\mathrm{SiO}_{2} / \mathrm{Si}$ substrate was cleaned with piranha solution (denoted $\mathrm{OH}$ ). The HKUST-1 concentration in the polymer solution was $2 \mathrm{mg} \mathrm{mL}{ }^{-1}$. The transfer characteristics of a HKUST-1/P3HT film spin-coated onto three different substrates were measured as the relative humidity increased from 0 to $30 \%$, after which the relative humidity was decreased to $0 \%$. In all devices, the drain current and threshold voltage increased as the relative humidity level increased, as shown in Fig. 3a.

In the hydrophilic $\mathrm{OH}$ substrate, the drain current at $V_{\mathrm{G}}=$ $20 \mathrm{~V}$ was $5.1 \times 10^{-8} \mathrm{~A}\left(I_{30} / I_{0}=80.9\right)$ and the threshold voltage
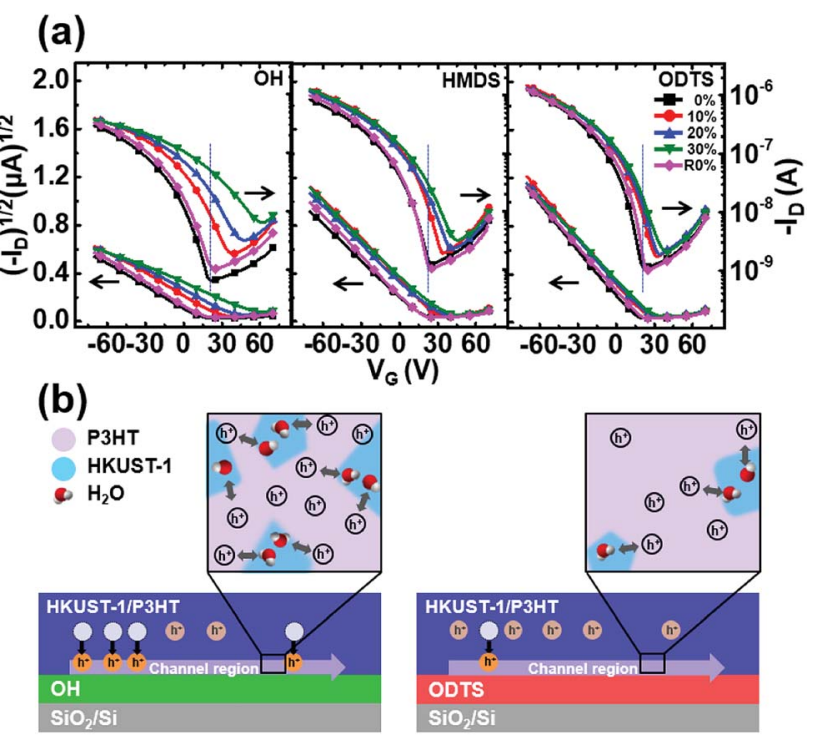

Fig. 3 (a) Transfer characteristics $\left(I_{D}-V_{G}\right)$ of a HKUST-1/P3HT hybrid film coated onto different substrates: OH, HMDS, and ODTS substrates at a fixed drain voltage $\left(V_{D}=-60 \mathrm{~V}\right)$ and various relative humidities, from $0 \%$ to $30 \%$. All HKUST-1/P3HT films were prepared with identical concentration of the solution, $2 \mathrm{mg} \mathrm{mL}^{-1}$ HKUST-1 in $10 \mathrm{mg} \mathrm{mL}^{-1}$ P3HT solution. (b) Schematic diagrams of the sensing mechanisms on the substrates: $\mathrm{OH}$ (left) and ODTS (right) substrate. shifted by $60 \mathrm{~V}$, high values compared to the corresponding values obtained from a hydrophobic ODTS-treated substrate, $1.4 \times 10^{-8} \mathrm{~A}\left(I_{30} / I_{0}=13\right)$ and $11 \mathrm{~V}$, respectively, at a relative humidity of $30 \%$ (see Table 2 ). The HMDS-treated substrate displayed intermediate values of $2.5 \times 10^{-8} \mathrm{~A}$ and $22 \mathrm{~V}$.

The transfer characteristics varied as a function of the relative humidity due to the generation and attraction of holes derived from the presence of water molecules in the HKUST-1 active layer. Contact between the oxidizing molecules, including $\mathrm{H}_{2} \mathrm{O}$, and the p-type semiconductor led to a doping process, ${ }^{37}$ and contact between $\mathrm{H}_{2} \mathrm{O}$ molecules and $\mathrm{P} 3 \mathrm{HT}$ generated holes in the semiconducting layer. In this study, the charge carrier concentration in the semiconducting layer was enhanced and the turn-on voltages of the devices shifted toward positive values as the relative humidity increased. A positive turn-on voltage from a p-type transistor indicates hole doping. At a high negative gate voltage, the drain current increased to a small degree due to the influence of the high field effect induced by the high gate voltage. The $\mathrm{H}_{2} \mathrm{O}$ molecules caused incomplete depletion in the OTFTs.

In a p-type organic semiconductor operated at a positive gate voltage $\left(V_{\mathrm{G}}>0\right)$, the hole carriers are pushed out from the interface between the dielectric layer and the semiconducting layer. In other words, only a low concentration of mobile holes is present in the channel region (the main charge carrier pathway), thereby forming a depletion mode in OTFTs. In the depletion mode, OTFTs display a very low current level, and the threshold voltage is close to $0 \mathrm{~V}$; however, in the HKUST-1/P3HT hybrid film, the hole carriers were not completely depleted due to the presence of $\mathrm{H}_{2} \mathrm{O}$ molecules in the MOFs. The large dipole moment of $\mathrm{H}_{2} \mathrm{O}(1.94 \mathrm{D})$ trapped holes in the vicinity of the active layer and prevented the number of mobile charge carriers in the active layer from decreasing significantly. The enrichment of charge carriers at the interface between the dielectric and semiconducting layer increased the drain current at a high positive gate voltage and shifted the threshold voltage toward positive values. ${ }^{38,39}$ Consequently, the large dipole moment of $\mathrm{H}_{2} \mathrm{O}$ acted as a doping process to create imperfect depletion in the device, thereby changing the hole carrier density in the channel region.

The transfer characteristics of the $\mathrm{OH}$ and ODTS substrates differed due to the positions of the MOFs embedded in the polymer film (see Fig. 3b). In a hydrophilic substrate, such as 
Table 2 Device performances of the HKUST-1/P3HT hybrid film prepared on three different substrates: OH, HMDS and ODTS

\begin{tabular}{llllrl}
\hline Substrate & $\mu_{\mathrm{RH} 0 \%}{ }^{a}\left(\mathrm{~cm}^{2} \mathrm{~V}^{-1} \mathrm{~s}^{-1}\right)$ & $\mu_{\mathrm{RH} 30 \%}{ }^{b}\left(\mathrm{~cm}^{2} \mathrm{~V}^{-1} \mathrm{~s}^{-1}\right)$ & $\operatorname{Drain~current}^{c}(\mathrm{~A})$ & $\Delta V_{\mathrm{th}}{ }^{d}(\mathrm{~V})$ \\
\hline OH & $3.2 \times 10^{-4}$ & $2.1 \times 10^{-4}$ & $5.1 \times 10^{-8}$ & 60 \\
HMDS & $1.0 \times 10^{-3}$ & $9.4 \times 10^{-4}$ & $2.5 \times 10^{-8}$ & 80.9 \\
ODTS & $1.3 \times 10^{-3}$ & $1.2 \times 10^{-3}$ & $1.4 \times 10^{-8}$ & 11 & 20.0 \\
\end{tabular}

${ }^{a}$ Average field-effect mobility calculated in the saturation regime at $0 \%$ relative humidity. ${ }^{b}$ Average field-effect mobility calculated in the saturation regime at $30 \%$ relative humidity. ${ }^{c}$ Drain current at $V_{\mathrm{G}}=20 \mathrm{~V} .{ }^{d}$ Variations in the threshold voltage shift. ${ }^{e}$ Current ratio of $I_{\mathrm{D}}$ at $30 \%$ compared to $I_{\mathrm{D}}$ at a relative humidity of $0 \%$, at $V_{\mathrm{G}}=20 \mathrm{~V}$.

the $\mathrm{OH}$ substrate, HKUST-1s were located near the bottom substrate, whereas HKUST-1 in the ODTS substrate were mostly far from the hydrophobic substrate. Because HKUST-1 in the semiconducting layer adsorbed a great number of $\mathrm{H}_{2} \mathrm{O}$ molecules due to its remarkable surface area $\left(2065 \mathrm{~m}^{2} \mathrm{~g}^{-1}\right.$ for the synthesized HKUST-1), the positions of the adsorbed $\mathrm{H}_{2} \mathrm{O}$ molecules were affected by the locations of the MOFs, which predominantly altered the electrical current.

The surface energy of HKUST-1 is reported to be $25.12 \mathrm{~mJ}$ $\mathrm{m}^{-2}$, the value obtained from a vacant open coordination site in HKUST-1. ${ }^{40}$ We modified the open coordination site of HKUST-1 by filling it with $\mathrm{H}_{2} \mathrm{O}$ molecules, and the surface energy of the modified HKUST-1 was expected to increase. The surface energy of P3HT is known to be $19.90 \mathrm{~mJ} \mathrm{~m}^{-2}$, relatively hydrophobic compared to the surface energy of HKUST-1. ${ }^{41}$ We observed a decrease in the water contact angles of the hybrid films as the HKUST-1 content increased due to the high surface energy of HKUST-1 (see Fig. S5†). In the hydrophilic substrate, HKUST-1 molecules were embedded near the interface between the dielectric layer and the semiconducting layer; that is, water molecules were located near the interface. Because the dielectric/semiconducting interface is a channel region that facilitates charge carrier transport, this effect enhanced the charge carrier concentration. Especially in the depletion mode, water molecules in the channel region stabilized the hole carriers by a factor of 80.9 , with a high off-state current, and the threshold voltage shift was large $\left(\Delta V_{\text {th }}=60 \mathrm{~V}\right)$. On the other hand, in the hydrophobic substrate, HKUST-1 molecules were located away from the dielectric-semiconducting interface. Only a few water molecules were located in the channel region. This feature did not significantly affect the drain current or the threshold voltage shift $\left(\Delta V_{\mathrm{th}}=11 \mathrm{~V}\right)$. Modifications to the hydrophilicity/hydrophobicity of the substrate can control the number of MOFs present in the charge carrier pathway, and this is useful for improving the sensing properties using a small amount of MOFs.

Fig. 4a shows an energy dispersive spectrometer (EDS) mapping image of a hybrid film. A Cu element with a violet color was observed to be more abundant at the thin film as the hydrophilicity of the substrate increased. $\mathrm{Cu}$ is present in the structure of HKUST-1, and a violet color indicates the presence of HKUST-1. The point EDS data presented in Fig. S6 $\dagger$ shows that $\mathrm{C}, \mathrm{O}, \mathrm{Cu}$, and $\mathrm{S}$ elements were present. $\mathrm{S}$ and $\mathrm{Cu}$ indicated the presence of P3HT and HKUST-1, respectively. The weight percent (atomic percent) of $\mathrm{Cu}$ on the $\mathrm{OH}$ substrate was $0.28 \%$ $\pm 0.15(0.06 \%)$, whereas it was $0.23 \% \pm 0.1(0.05 \%)$ on the HMDS substrate and $0.20 \% \pm 0.08(0.04 \%)$ on the ODTS substrate. To explain the different amount of HKUST-1 in three substrates, the thickness values of the spin-coated hybrid films were determined by Lambert's law from UV-Vis spectra and ellipsometer (see Fig. S7 $\dagger$ ). Although the same concentration of the solution ( $2 \mathrm{mg} \mathrm{mL} \mathrm{mL}^{-1}$ HKUST-1 to $10 \mathrm{mg} \mathrm{mL}^{-1}$ of P3HT solution) was coated onto the three different substrates, thickness of the hybrid film decreased with increasing hydrophobicity of the substrate: $92 \mathrm{~nm}$ on $\mathrm{OH}, 86 \mathrm{~nm}$ on HMDS, $76 \mathrm{~nm}$ on ODTS substrate. The hydrophobic substrate pushed out the solution during the spinning process, which results in the decrease of the P3HT film thickness and amount of HKUST-1.

X-ray photoelectron spectroscopy (XPS) was used to investigate the compositional gradient of HKUST- 1 in the P3HT films as a function of the film depth. ${ }^{42}$ Fig. $4 \mathrm{~b}$ compares the compositions of HKUST-1 in the P3HT films near a buried interface, using XPS measurements. HKUST-1/P3HT hybrid films were flipped over by detaching the film from each substrate using epoxy adhesive, which results in an atomic analysis on the

(a)

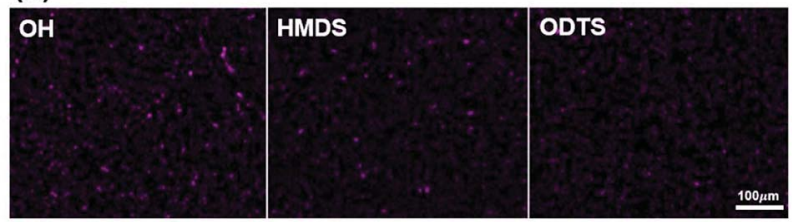

(b)

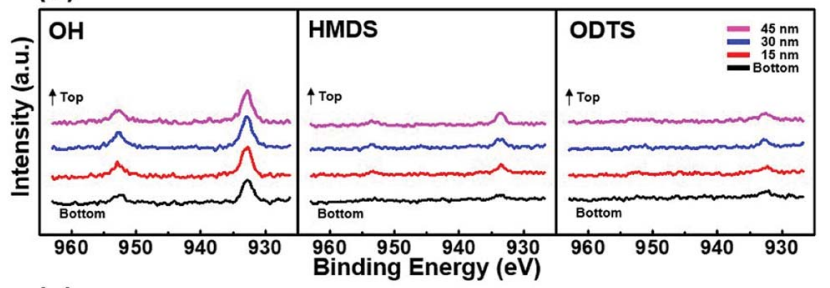

(c)
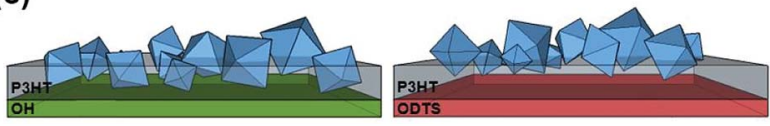

Fig. 4 (a) Energy dispersive spectrometer mapping image of the hybrid film, which had been flipped over by detaching the hybrid film from each substrate using an epoxy adhesive. (b) XPS depth profile of $\mathrm{Cu} 2 \mathrm{p}$ along the bottom side of the hybrid film, obtained by sputtering with an Ar ion gun. As the film was etched by Ar for 1 min, the thickness of the film decreased by $15 \mathrm{~nm}$. The flipped HKUST-1/P3HT film was prepared by detaching thin films from the substrates: $\mathrm{OH}, \mathrm{HMDS}$, and ODTS substrates. Hybrid film was equally prepared with $2 \mathrm{mg}$ of HKUST-1 added $10 \mathrm{mg} \mathrm{mL}^{-1}$ of P3HT solution. (c) Schematic illustrations showing the distributions of HKUST-1 in the P3HT films prepared on the different substrates: $\mathrm{OH}$ (left) and ODTS (right) substrate. 
bottom region of the film. Hybrid films delaminated from three different substrates were sputtered using an Ar ion gun to eliminate the film. ${ }^{43}$ As the film was etched for $1 \mathrm{~min}$, the thickness of the film decreased by about $15 \mathrm{~nm}$. The Cu signal, which corresponded to HKUST-1, was collected at each thickness and is shown in Fig. 4b. ${ }^{\mathbf{4 4 , 4 5}}$ The HKUST-1/P3HT film coated onto a $\mathrm{OH}$ substrate revealed a $\mathrm{Cu}$ signal with a high value, whereas the film coated on HMDS or ODTS substrates revealed a $\mathrm{Cu}$ signal with a very small value near the buried interface. These results confirmed that the surface energy strongly induced vertical phase separation between HKUST-1 and P3HT during the coating process. The hybrid film coated onto an ODTS substrate displayed a negligible $\mathrm{Cu}$ peak at the bottom region near the substrate. Fig. $4 \mathrm{c}$ shows a schematic diagram of a hybrid film on the different substrates. The polarity of the hydrophilic substrate attracted polar materials, whereas repulsive forces worked on the non-polar (hydrophobic) materials. $^{\mathbf{4 6 , 4 7}}$ The hydrophilic HKUST-1 on the $\mathrm{OH}$ substrate preferred locations close to the buried interface, unlike the ODTS substrate, in which HKUST-1 was embedded far from the substrate due to repulsion with the hydrophobic ODTS. The result show that the physical properties of the substrate relocated HKUST-1 within the polymer film and affected the electrical characteristics of the OTFT-based sensor. Although the content of MOFs was not totally same on the three modified substrates, XPS data shows that the amount of HKUST-1 in the bottom region on $\mathrm{OH}$ substrate was more abundant than that of ODTS substrate, which result in the charge carrier enhancement in the channel region.

Finally, the gas sensing properties of the HKUST-1/P3HT hybrid film coated onto an HMDS substrate were measured, as shown in Fig. 5. The transfer characteristics were measured as the humidity levels was adjusted cyclically between $0 \%$ and $30 \%$. During the measurements collected at high and low humidity levels, the sensors displayed reliable performances with good agreement in both the current level and the turn-on voltage (see Fig. 5a). The current level at $V_{\mathrm{G}}=25 \mathrm{~V}$ was extracted from the transfer curve shown in Fig. 5b. The humidity level underwent 50 cycles with very little variation in the device response, indicating stable transfer characteristics. The sensing dynamics, shown in Fig. $5 \mathrm{c}$, were measured at $V_{\mathrm{G}}=25 \mathrm{~V}$ and $V_{\mathrm{D}}=-60 \mathrm{~V}$, and the humidity was cycled between $0 \%$ and $30 \%$. The sensor responded very rapidly to the sensor, with a $0.23 \mathrm{~s}$ response time and a $2.91 \mathrm{~s}$ recovery time (see Fig. $5 \mathrm{~d}$ ). The sensor provided a tenfold slower recovery time compared to the response time due to the slow desorption of water molecules from the MOFs. However, the recovery time is still fast compare to most other OTFT based gas sensors which require hundreds of second to recover..$^{\mathbf{7 1 8 , 4 8 , 4 9}}$ This study shows that the MOF/polymer hybrid film achieved excellent sensing abilities in terms of sensitivity and stability compared to conventional polymer-based films due to the outstanding gas adsorption properties of MOFs.

\section{Conclusions}

A hybrid film consisting of a conjugated polymer (P3HT), along with MOFs (HKUST-1) was fabricated for use in stable (a)

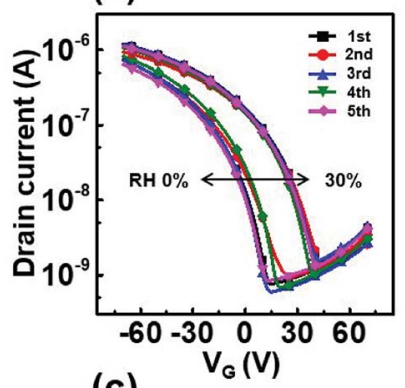

(c)

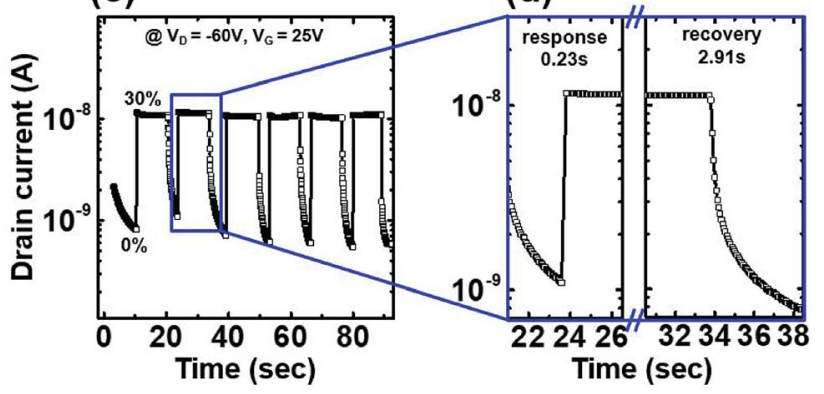

Fig. 5 (a) Transfer characteristics of hybrid film coated onto HMDS substrate with $2 \mathrm{mg}$ of HKUST-1 added $10 \mathrm{mg} \mathrm{mL}^{-1}$ of P3HT solution over the humidity level range, between 0 and $30 \%$, under repeated cycles. (b) Plot of the drain current at $V_{D}=-60 \mathrm{~V}$ and $V_{G}=25 \mathrm{~V}$ as a function of the switching cycle number. (c) Sensing dynamics and (d) magnified view across the relative humidity values between 0 and $30 \%$ at $V_{D}=-60 \mathrm{~V}$ and $V_{G}=25 \mathrm{~V}$

OTFT-based gas sensors. An organic/inorganic hybrid material with a high surface area and gas adsorbing properties was introduced into the conjugated polymer, and target gas was effectively attracted to the active layer on the OTFT, significantly altering the transfer characteristics. Water molecules were attracted to the hybrid active layer, leading to a doping process and creating an imperfect depletion mode in the device. Water doping enriched the channel region with hole carriers, increased the drain current, and shifted the threshold voltage. The physical characteristics of each material and the chemical modifications to the substrate permitted control over vertical phase separation, thereby improving the sensitivity of the gas sensor. These results suggested that the organic/ inorganic hybrid materials may be useful in sensitive, flexible, lightweight and selective polymeric gas sensors applicable to a variety of gases beyond water.

\section{Conflicts of interest}

There are no conflicts to declare.

\section{Acknowledgements}

This research was supported by Basic Science Research Program through the National Research Foundation of Korea (NRF) funded by the Ministry of Education (2016R1D1A1B03931906). 


\section{References}

1 Y. D. Park, J. A. Lim, H. S. Lee and K. Cho, Mater. Today, 2007, 10, 46-54.

2 P. Lin and F. Yan, Adv. Mater., 2012, 24, 34-51.

3 B. Kumar, B. K. Kaushik and Y. S. Negi, Polym. Rev., 2014, 54, 33-111.

4 Z. Liu, L. Zhang, S. Poyraz and X. Zhang, Curr. Org. Chem., 2013, 17, 2256-2267.

5 X. Zhao and Z. Zhan, Chem. Soc. Rev., 2010, 40, 3728-3743.

6 C. D. Dimitrakopoulos and D. J. Mascaro, IBM J. Res. Dev., 2001, 45, 11-27.

7 T. Xie, G. Xie, H. Du, Y. Su, Z. Ye, Y. Chen and Y. Jiang, Sens. Actuators, B, 2016, 230, 176-183.

8 M. R. Cavallari, J. E. E. Izquierdo, G. S. Braga, E. A. T. Dirani, M. A. Pereira-da-Silva, E. F. G. Rodríguez and F. J. Fonseca, Sensors, 2015, 15, 9592-9609.

9 X. Guo, Y. Xu, S. Ogier, T. N. Ng, M. Caironi, A. Perinot, L. Li, J. Zhao, W. Tang, R. A. Sporea, A. Nejim, J. Carrabina, P. Cain and F. Yan, IEEE Trans. Electron Devices, 2017, 64, 1906-1921.

10 D. Elkington, N. Cooling, W. Belcher, P. C. Dastoor and X. Zhou, Electronics, 2014, 3, 234-254.

11 S. Khan, L. Lorenzelli and R. S. Dahiya, IEEE Sens. J., 2015, 15, 3164-3185.

12 L. Zhang, W. Du, A. Nautiyal, Z. Liu and X. Zhang, Sci. China Mater., 2018, 61, 303-352.

13 Rajesh, T. Ahuja and D. Kumar, Sens. Actuators, B, 2009, 136, 275-286.

14 S. H. Yu, J. Cho, K. M. Sim, J. U. Ha and D. S. Chung, ACS Appl. Mater. Interfaces, 2016, 8, 6570-6576.

15 L. Qiu, W. H. Lee, X. Wang, J. S. Kim, J. A. Lim, D. Kwak, S. Lee and K. Cho, Adv. Mater., 2009, 21, 1349-1353.

16 B. Nketia-Yawson, A.-R. Jung, Y. Noh, G.-S. Ryu, G. D. Tabi, K.-K. Lee, B. Kim and Y.-Y. Noh, ACS Appl. Mater. Interfaces, 2017, 9, 7322-7330.

17 B. H. Lee, G. C. Bazan and A. J. Heeger, Adv. Mater., 2016, 28, 57-62.

18 Y. Seo, J. H. Lee, J. E. Anthony, K. V. Nguyen, Y. H. Kim, H. W. Jang, S. Ko, Y. Cho and W. H. Lee, Adv. Mater. Interfaces, 2017, 5, 1701399.

19 C. Zhang, P. Chen and W. Hu, Chem. Soc. Rev., 2015, 44, 2087-2107.

20 Q. Wang, S. Wu, F. Ge, G. Zhang, H. Lu and L. Qiu, Adv. Mater. Interfaces, 2016, 3, 1600518.

21 R. Ahmed, A. Kadashchuk, C. Simbrunner, G. Schwabegger, M. A. Baig and H. Sitter, ACS Appl. Mater. Interfaces, 2014, 6, 15148-15153.

22 H. Tai, X. Li, C. Duan, G. Xie and Y. Jiang, Integr. Ferroelectr., 2014, 153, 65-72.

23 F. A. A. Paz, J. Klinowski, S. M. F. Vilela, J. P. C. Tomé, J. A. S. Cavaleiro and J. Rocha, Chem. Soc. Rev., 2012, 41, 1088-1110.

24 P. Silva, S. M. F. Vilela, J. P. C. Tomé and F. A. A. Paz, Chem. Soc. Rev., 2015, 44, 6774-6803.
25 H. Wang, J. Peng and J. Li, Chem. Rec., 2016, 16, 1298-1310. 26 H. Furukawa, K. E. Cordova, M. O'Keeffe and O. M. Yaghi, Science, 2013, 341, 1230444.

27 B. Liu, J. Mater. Chem., 2012, 22, 10094-10101.

28 M. Rieger, M. Wittek, P. Scherer, S. Löbbecke and K. MüllerBuschbaum, Adv. Funct. Mater., 2018, 28, 1704250.

29 J. Park, H. Lee, Y. E. Bae, K. C. Park, H. Ji, N. C. Jeong, M. H. Lee, O. J. Kwon and C. Y. Lee, ACS Appl. Mater. Interfaces, 2017, 9, 28758-28765.

30 P. Küsgens, M. Rose, I. Senkovska, H. Fröde, A. Henschel, S. Siegle and S. Kaskel, Microporous Mesoporous Mater., 2009, 120, 325-330.

31 G. Lu, O. K. Farha, L. E. Kreno, P. M. Schoenecker, K. S. Walton, R. P. V. Duyne and J. T. Hupp, Adv. Mater., 2011, 23, 4449-4452.

32 Z. Liang, M. Marshall and A. L. Chaffee, Energy Fuels, 2009, 23, 2785-2789.

33 K.-S. Lin, A. K. Adhikari, C.-N. Ku, C.-L. Chiang and H. Kuo, Int. J. Hydrogen Energy, 2012, 37, 13865-13871.

34 Z. Zhu, J. T. Mason, R. Dieckmann and G. G. Malliaras, Appl. Phys. Lett., 2002, 81, 4643-4645.

35 T. Mori, Y. Kikuzawa and K. Noda, J. Appl. Phys., 2013, 52, $05 \mathrm{DC} 02$.

36 S. Wu, G. Wang, Z. Xue, F. Ge, G. Zhang, H. Lu and L. Qiu, ACS Appl. Mater. Interfaces, 2017, 9, 14974-14982.

37 S. Hoshino, M. Yoshida, S. Uemura, T. Kodzasa, N. Takada, T. Kamata and K. Yase, J. Appl. Phys., 2004, 95, 5088-5093.

38 S. H. Kim, H. Yang, S. Y. Yang, K. Hong, D. Choi, C. Yang, D. S. Chung and C. E. Park, Org. Electron., 2008, 9, 673-677.

39 J. Park, J.-H. Bae, W.-H. Kim, M.-H. Kim, C.-M. Keum, S.-D. Lee and J. S. Choi, Materials, 2010, 3, 3614-3624.

40 S. Amirjalayer, M. Tafipolsky and R. Schmid, J. Phys. Chem. Lett., 2014, 5, 3206-3210.

41 J. B. Howard, S. Noh, A. E. Beier and B. C. Thompson, ACS Macro Lett., 2015, 4, 725-730.

42 H.-S. Kang, M. S. P. Reddy, D.-S. Kim, K.-W. Kim, J.-B. Ha, Y. S. Lee, H.-C. Choi and J.-H. Lee, J. Phys. D: Appl. Phys., 2013, 46, 155101.

43 S. Rtimi, Catalysts, 2017, 7, 57.

44 H. Chen, L. Wang, J. Yang and R. T. Yang, J. Phys. Chem. C, 2013, 117, 7565-7576.

45 J. Li, Z. Mei, L. Liu, H. Liang, A. Azarov, A. Kuznetsov, Y. Liu, A. Ji, Q. Meng and X. Du, Sci. Rep., 2014, 4, 7240.

46 M. Kim, J.-H. Kim, H. H. Choi, J. H. Park, S. B. Jo, M. Sim, J. S. Kim, H. Jinnai, Y. D. Park and K. Cho, Adv. Energy Mater., 2014, 4, 1300612.

47 M. Chang, D. Choi, G. Wang, N. Kleinhenz, N. Persson, B. Park and E. Reichmanis, ACS Appl. Mater. Interfaces, 2015, 7, 14095-14103.

48 Y. D. Park, B. Kang, H. S. Lim, K. Cho, M. S. Kang and J. H. Cho, ACS Appl. Mater. Interfaces, 2013, 5, 8591-8596.

49 K. Manoli, L. M. Dumitru, M. Y. Mulla, M. Magliulo, C. D. Franco, M. V. Santacroce, G. Scamarcio and L. Torsi, Sensors, 2014, 14, 16869-16880. 